\title{
The Research on Ticket Fare Optimization for China's High-Speed Train
}

\author{
Jinzi Zheng and Jun Liu \\ School of Traffic and Transportation, Beijing Jiaotong University, Beijing 100044, China \\ Correspondence should be addressed to Jinzi Zheng; 10114214@bjtu.edu.cn
}

Received 6 June 2016; Accepted 28 July 2016

Academic Editor: Paolo Crippa

Copyright ( 2016 J. Zheng and J. Liu. This is an open access article distributed under the Creative Commons Attribution License, which permits unrestricted use, distribution, and reproduction in any medium, provided the original work is properly cited.

\begin{abstract}
With the constant deepening of the railway reform in China, the competition between railway passenger transportation and other modes of passenger transportation has been getting fiercer. In this situation, the existing unitary and changeless fare structure gradually becomes the prevention of railway revenue increase and railway system development. This paper examines a new method for ticket fare optimization strategy based on the revenue management theory. On the premise that only one fare grade can be offered for each OD at the same time, this paper addresses the questions of how to determine the number of fare grades and the price of each fare grade. First, on the basis of piecewise pricing strategy, we build a ticket fare optimization model. After transforming this model to a convex program, we solve the original problem by finding the Kuhn-Tucker (K-T) point of the convex program. Finally, we verify the proposed method by real data of Beijing-Shanghai HSR line. The calculating result shows that our pricing strategy can not only increase the revenue, but also play a part in regulating the existing demand and stimulating potential demand.
\end{abstract}

\section{Introduction}

With the constant deepening of the railway reform in China, railway passenger transportation has been involved in fiercer competition with airline and highway transportation. In the environment of market economy, the primary goal of railway sector is not only to realize passengers' transportation demand, but also to get as much revenue as possible. However, in the past several decades, what we have been applying is the unitary and changeless fare structure, of which the ticket fare rate is the same all over the country and for all kinds of passengers. Statistical data provided for 2014 by the Beijing-Shanghai High-Speed Railway Company shows that 13 percent of the trains had a load factor less than 65 percent, which means there exists large potential of revenue in railway passenger transportation. It is hard for the railway to sustain the position as before if we do not make any transition to adapt to the new situation. Since 2012, the Shanghai-Nanjing, Shanghai-Hangzhou, BeijingShanghai, and Beijing-Guangzhou high-speed railways have tried to discount off-season ticket fares. One characteristic of these preference strategies is that the timing and discount level are the same for all the ODs of a specific train. This unitary discount strategy is easy to be implemented but cannot accommodate to diversified demand characteristics (such as demand density and demand valuation) of various ODs in different time.

Revenue management, which has been applied to air transportation for years, has successfully increased the revenue of air transportation by providing proper customers with proper prices and effective capacity control strategies. General and detailed discussion of revenue management can be seen in Weatherford and Bodily [1], McGill and Van Ryzin [2], Bitran and Caldentey [3], and Elmaghraby and Keskinocak [4]. Since railway passenger transportation has many characters in common with air transportation, we are confident that revenue management can also bring benefit to railway if being utilized properly. Armstrong and Meissner [5] analyzed the similarities and differences between airline and railway passenger transportation and gave an overview of the existing research results on railway revenue management.

A number of previous works have examined the aspects of the problem of price-based revenue management, which is aimed at increasing profits by dynamically setting optimal pricing strategies [6]. Dynamic pricing has already been successfully applied in many industries such as airline, hotel, 
and car rental. Kincaid and Darling [7] laid the foundation for the study of multigrade price, building two optimization models to generate the optimal prices of each time from the aspects of whether the price is announced. Chatwin [8] researched how to choose the optimal prices from a given price set according to the current inventory. Gallego and Ryzin [9] analyzed the structural characteristics of optimal price for a single product, proving that the optimal solution of the deterministic problem is the upper bound of the stochastic problem. On this basis, Gallego and Van Ryzin [10] found the upper bound of the optimal expected revenue by analyzing the deterministic version of the problem for multiple products. Pricing belongs to a tactical problem. On the practical level, methods are needed to dynamically decide the optimal timing of price changes. For a unique price change allowed to be either higher or lower, Feng and Gallego [11] put forward a control strategy based on a time threshold that depends on the number of unsold items.

In China, research on dynamic pricing started late because the ticket price has traditionally remained changeless. From the practical point of view, Shi [12] translated the optimal solution to a feasible approximate optimal solution. Zhang [13] applied maximum concave envelope theory to determine the optimal fare discount for each OD of a train. With the application of dynamic game theory, Zhang et al. [14] established a dynamic pricing model between HSR and air transportation. Yao et al. [15] analyzed the pricing strategy for HSR in Wuhan-Guangzhou corridor with the consideration of the competition between passenger rail transportation and other transport modes. Bingyi [16] developed dynamic programming model to deal with the HSR revenue optimization problem with multitrain, multisegment, and multiclass, by considering passengers choice behavior.

Our paper studies the ticket fare optimization method for single high-speed train, aiming at optimizing the revenue of the train. A train service is represented as a linear network with stations as nodes and arcs that connect OD station pairs served. We propose a method to determine the number of fare grades according to the regularity of passenger arrival and the optimal prices that meet the demand of each OD. A demand driven fare is allowed to fluctuate within a certain range, either upward or downward, around the standard fare.

This paper proceeds as follows. Section 2.1 is a fine description of optimization model. Section 2.2 makes use of Lagrange Multiplier and Karush-Kuhn-Tucker (KKT) conditions to solve the previous model. Section 3 shows an experimental study for verifying the proposed method.

\section{Model and Algorithm}

We will firstly provide in this section the basic terminologies and notations necessary for modeling. After that, the model and algorithm of ticket fare optimization problem are put forward.

2.1. The Notations and Model. We define the "product" in our study as origin-destination (OD) transportation service offered by a passenger train, which contains $M+1$ stops including the starting and terminal stations and $M$ sections between each two stations. The product in our model is denoted by $(i j)=\{(i j): j>i ; i=1, \ldots, M ; j=2, \ldots, M+$ 1 , referring to the OD transportation service from station $i$ to station $j$. All the products produced by a passenger train are stored in a sequence according to the incremental order of $j$ and $i,(I J)=\{(12), \ldots,(1(M+1)),(23), \ldots,(M(M+1))\}$. After numbering all the products in $(I J)$ from left to right, we can get a new product sequence indexed by the serial number, $b=\left(b^{1}, \ldots, b^{N}\right)$, and $N=M(M+1) / 2$. The section, which is a basic element to make up OD, is deemed as the resource of product, denoted as $m=1, \ldots, M$.

By analyzing the historical ticket-sale data, we found the demand density for a certain OD product varies with time regularly in ticket-sale period. As we deem, the passenger ticket-purchasing behavior is influenced by various factors, the details on which will be discussed in Section 3.1. For a certain product, the external factors such as travel distance, alternative product, and departure time are fixed; thus the difference of ticket-purchasing pattern by time is mainly determined by the passengers' valuations. As is usually done in airline revenue management, we assume that passengers with low valuation arrive earlier and those with high valuation arrive later. Based on the third-degree price discrimination, profit could be increased by providing different customers with corresponding prices. Therefore, the idea of floating pricing strategy proposed here is to set prices separately by product, and for a certain product the price is adjusted according to the change of passenger arrival pattern.

We firstly divide the ticket-sale period into several subperiods according to the statistical regularity of passenger arrival process of the product we concern and then set the price in each subperiod. For an OD product, the ticket-sale period is divided into $K$ subperiods; the $k$ th one is denoted by $t_{k}$. The time duration of subperiod $t_{k}$ of product $n$ is $l_{n}\left(t_{k}\right)$. In this research, we make the following hypotheses: (1) the passenger transportation market is an imperfect competition market and the demand of a certain product changes with its price; (2) we only consider myopic passengers who buy tickets as soon as the offered price is less than their willingness to pay, in contrast to the strategic passengers who will optimize their purchase behavior by predicting the price strategies; (3) for simplification, we assume there is only one class of seats on the train. Thus, the demand density of product $n$ in subperiod $t_{k}$ can be expressed as the function of $t_{k}$ and current price $p_{n}\left(t_{k}\right)$, denoted as $\lambda_{n}\left(t_{k}, p_{n}\left(t_{k}\right)\right)$. We control demand density instead of price in this model for the convenient description of capacity constraint. The price of product is denoted as the inverse function of demand function, $p(t, \lambda)$. To ensure the price is feasible, we define a feasible price set $\mathscr{P}$. The decision variables in the model are demand densities of each product in each subperiod, $\lambda=\left(\lambda_{1}\left(t_{1}\right), \ldots, \lambda_{1}\left(t_{K}\right), \lambda_{2}\left(t_{1}\right), \ldots, \lambda_{N}\left(t_{K}\right)\right)$ where $\lambda \in \Lambda(\Lambda=\{\lambda(t, p): p \in \mathscr{P}\})$.

In our problem, the initial inventory is expressed as a vector $x=\left(x^{1}, \ldots, x^{M}\right)^{T} \in \mathfrak{I}_{+}^{M}\left(\mathfrak{I}_{+}\right.$is the set of positive integers), where $x^{m}$ is the initial inventory of resource $m$, representing the number of available seats of this section. In addition, we define a $0-1$ variable $a_{m n}$ to denote the relationship 
between product $n$ and resource $m$; that is, if product $n$ occupies resource $m, a_{m n}=1$; else $a_{m n}=0$. The preallocation plan is a peculiarity of railway passenger organization in China, which is to set the range of application for all seats ahead of the ticket-sale period. In our model, $y^{n}$ is used to denote the maximum capacity of product $n$ produced by the preallocation plan, which is another important capacity constraint.

The total revenue of the train we concern is as below:

$$
R(\lambda, t)=\sum_{n=1}^{N} \sum_{k=1}^{K} l_{n}\left(t_{k}\right) \cdot \lambda_{n}\left(t_{k}\right) \cdot p_{n}\left(\lambda_{n}\left(t_{k}\right)\right) .
$$

The optimal price strategy is the one $p^{*}\left(\lambda^{*}\right.$ in this model) that will maximize the total revenue $R^{*}\left(\lambda^{*}, t\right)=$ $\max _{\lambda \in \Lambda} R(\lambda, t)$.

As stated above, we take into consideration two kinds of capacity constraints: one is the "resource capacity constraint", which ensures the capacity of any resource cannot be exceeded; the other is the "product capacity constraint" produced by preallocation plan. These constraints are expressed as (2) and (3), respectively:

$$
\begin{array}{r}
\sum_{n=1}^{N} \sum_{k=1}^{K} l_{n}\left(t_{k}\right) \cdot \lambda_{n}\left(t_{k}\right) \cdot a_{m n} \leq x^{m} \quad \forall m \in[1, M], \\
\sum_{k=1}^{K} l_{n}\left(t_{k}\right) \cdot \lambda_{n}\left(t_{k}\right) \leq y^{n} \quad \forall n \in[1, N] .
\end{array}
$$

2.2. The Algorithm. The revenue rate function $r(\lambda)=\lambda p(\lambda)$ is continuous, bounded, and concave [10]. As the product of real number and concave function and the sum of several concave functions are both concave, it is easy to deduce that the objective function $R(\lambda, t)$ is concave too. Therefore, the nonlinear programming problem we propose can be solved using Lagrange Multiplier and KKT conditions. According to the requirement of KKT condition, we introduce a new objective function $f(\lambda, t)$ which is the opposite of $R(\lambda, t)$. Then the initial problem can be rewritten as follows:

$$
\begin{aligned}
f(\lambda, t) & =-R(\lambda, t) \\
& =-\sum_{n=1}^{N} \sum_{k=1}^{K} l_{n}\left(t_{k}\right) \cdot \lambda_{n}\left(t_{k}\right) \cdot p_{n}\left(\lambda_{n}\left(t_{k}\right)\right), \\
g_{m}(\lambda, t)=x^{m}-\sum_{n=1}^{N} \sum_{k=1}^{K} l_{n}\left(t_{k}\right) \cdot \lambda_{n}\left(t_{k}\right) \cdot a_{m n} \geq 0 & \forall m \in[1, M], \\
h_{n}(\lambda, t)=y^{n}-\sum_{k=1}^{K} l_{n}\left(t_{k}\right) \cdot \lambda_{n}\left(t_{k}\right) \geq 0 & \forall n \in[1, N] .
\end{aligned}
$$

The gradient of the objective function and constraint functions can be gained by taking the derivation:

$$
\begin{aligned}
& \nabla f(\lambda)=-\left[l_{1}\left(t_{1}\right) p_{1}\left(\lambda_{1}\left(t_{1}\right)\right), \ldots, l_{1}\left(t_{K}\right)\right. \\
& \cdot p_{1}\left(\lambda_{1}\left(t_{K}\right)\right), l_{2}\left(t_{1}\right) p_{2}\left(\lambda_{2}\left(t_{1}\right)\right), \ldots, l_{N}\left(t_{K}\right)
\end{aligned}
$$

$$
\begin{gathered}
\left.\cdot p_{N}\left(\lambda_{N}\left(t_{K}\right)\right)\right]^{T}, \\
\nabla g_{m}(\lambda)=-\left[l_{1}\left(t_{1}\right) a_{m 1}, \ldots, l_{1}\left(t_{K}\right) a_{m 1}, l_{2}\left(t_{1}\right)\right. \\
\left.\cdot a_{m 2}, \ldots, l_{N}\left(t_{K}\right) a_{m N}\right]^{T} \quad \forall m \in[1, M], \\
\nabla h_{n}(\lambda)=-[\underbrace{0, \ldots, 0}_{(n-1) K}, l_{1}\left(t_{1}\right) \lambda_{1}\left(t_{1}\right), \ldots, l_{1}\left(t_{K}\right)
\end{gathered}
$$

$$
\cdot \lambda_{1}\left(t_{K}\right), \underbrace{0, \ldots, 0}_{(N-n) K}]^{T} \quad \forall n \in[1, N] \text {. }
$$

Supposing that $\lambda^{*}$ is the optimal solution of the initial nonlinear programming problem (see (1)-(3)), and the derivation $\nabla g_{m}\left(\lambda^{*}\right)(m=1,2, \ldots, M)$ and $\nabla h_{n}\left(\lambda^{*}\right)(n=1,2$, $\ldots, N)$ are linearly independent, by introducing Lagrange multipliers $\mu^{*}=\left(\mu_{1}^{*}, \mu_{2}^{*}, \ldots, \mu_{M}^{*}\right)^{T}$ and $\rho^{*}=\left(\rho_{1}^{*}, \rho_{2}^{*}, \ldots\right.$, $\left.\rho_{N}^{*}\right)^{T}$, we can have

$$
\begin{aligned}
\nabla f\left(\lambda^{*}\right)-\sum_{m=1}^{M} \mu_{m}^{*} \nabla g_{m}\left(\lambda^{*}\right)-\sum_{n=1}^{N} \rho_{n}^{*} \nabla h_{n}\left(\lambda^{*}\right) & =0, \\
\mu_{m}^{*} g_{m}\left(\lambda^{*}\right) & =0, \\
m & =1,2, \ldots, M, \\
\rho_{n}^{*} h_{n}\left(\lambda^{*}\right) & =0, \\
n & =1,2, \ldots, N, \\
\mu_{m}^{*} & \geq 0, \\
m & =1,2, \ldots, M, \\
\rho_{n}^{*} & \geq 0, \\
n & =1,2, \ldots, N .
\end{aligned}
$$

By solving the above equations set we can get the optimal solution $\lambda^{*}$ and also the optimal price of each product in each subperiod.

\section{Case Study}

In this case study, we select two high-speed trains of BeijingShanghai High-Speed Railway G1 and G3, which have many things in common with each other in operation properties. Both of G1 and G3 trains depart from Beijing South station, stop once at Nanjing South station, and terminate at Shanghai Hongqiao station. The distance of Beijing-Nanjing product is $1023 \mathrm{~km}$, Beijing-Shanghai product $1318 \mathrm{~km}$, and NanjingShanghai product $295 \mathrm{~km}$. The single difference between these two trains is that G1 departs at 9:00 am and G3 at 2:00 pm.

3.1. Passenger Arrival Regularity. Passenger arrival regularity is the basis of segmenting the ticket-selling period. We apply the ticket-sale data in 2012 (the data of December is not 

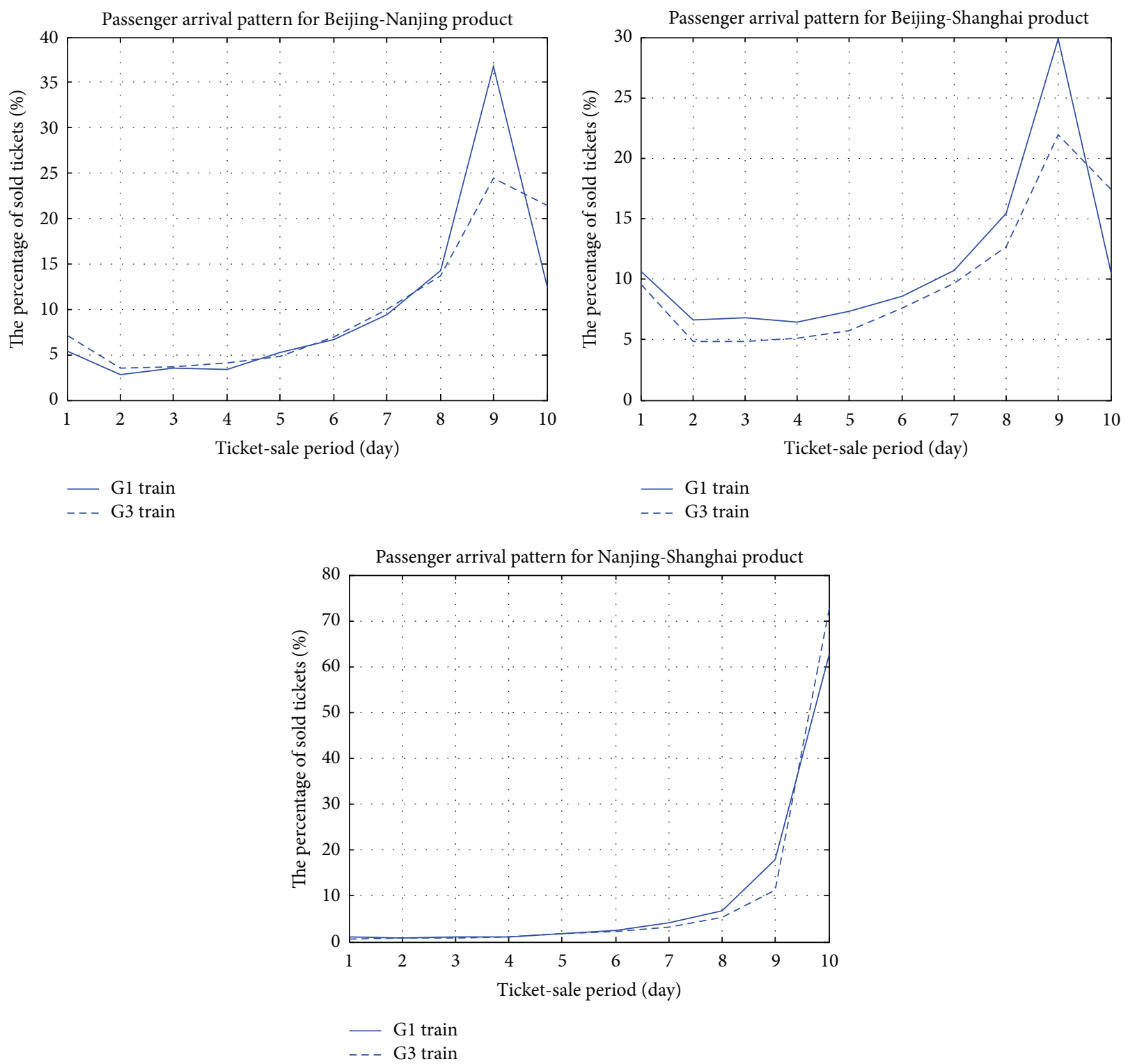

FIgURE 1: Passenger arrival regularity for G1 and G3 by product.

included for the lack of data in this month) as the sample to analyze these two trains' passenger arrival regularity by product and show the statistical results in Figure 1, of which each point represents the percentage of tickets sold in that day to the total amount of tickets sold in the entire period for the product.

In the figure, Day 1 is the first day of ticket-sale period, and Day 10 is the departure day. From Figure 1 we can see the passenger arrival regularity of Beijing-Nanjing product and that of Beijing-Shanghai product are similar to each other: from Day 1 to Day 8 the percentage of tickets sold per day stays low (15\% at most) and increases gradually; in Day 9 the percentage abruptly increases, and the ticket-sale rate per unit time peaks in Day 10 (dividing the amount of tickets sold in Day 10 by the actual selling time). The passenger arrival regularity of Nanjing-Shanghai product is special: in the first 9 days, the daily ticket-sale percent is very low and the climbing speed is low too; in Day 10 the percent is the biggest and much bigger than those of the first 9 days. From the above statistical results, we can conclude that passenger arrival regularity is affected by the following factors.

(1) Travel Distance. The longer the travel distance is, the earlier passengers tend to purchase tickets. Usually for the shortdistance travel, such as from Nanjing to Shanghai, passengers are inclined to purchase tickets just on the day of departure. As is shown in Figure 1, the percentage of passengers purchasing tickets in the earlier stage is the highest for BeijingShanghai product and lowest for Nanjing-Shanghai product.

To support this viewpoint, we did a data statistics using the historical ticket data for the Beijing-Shanghai High-Speed Railway during the period March 1, 2013, to March 13, 2014. 
TABLE 1: Regression analysis results.

\begin{tabular}{lccccccc}
\hline & Day 1 & Day 2 & Day 3 & Day 4 & Day 5 & Day 6 & Day 7 \\
\hline$a$ & 0.0104 & -0.0301 & -0.1274 & -0.0980 & -0.0631 & 0.0156 & 0.0304 \\
$b$ & 4.8397 & 0.8391 & 1.1323 & 0.9584 & 0.8424 & 0.5160 \\
$R$ square & 0.5645 & 0.8033 & 0.7561 & 0.7957 & 0.8152 & 0.6057 \\
Adjusted $R$ & 0.5373 & 0.7910 & 0.7408 & 0.7829 & 0.8036 & 0.5811 & 0.5474 \\
$P$ value & 0.0003 & 0.0044 & 0.0069 & 0.0060 & 0.0027 & 0.0001 & 0.0004 \\
\hline & Day 8 & Day 9 & Day 10 & Day 11 & Day 12 & Day 13 \\
\hline$a$ & 0.0297 & 0.0140 & 0.0346 & 0.1787 & 0.0630 & 0.1806 \\
$b$ & 0.8806 & 1.0221 & 1.1746 & 1.1391 & 1.4461 & 2.0868 \\
$R$ square & 0.7947 & 0.8240 & 0.7948 & 0.7938 & 0.8120 & 0.7486 \\
Adjusted $R$ & 0.7819 & 0.8130 & 0.7820 & 0.7810 & 0.8002 & 0.7329 \\
$P$ value & 0.0062 & 0.0018 & 0.0062 & 0.0065 & 0.0031 & 0.0088 \\
\hline & Day 15 & Day 16 & Day 17 & Day 18 & Day 19 & 0.9067 \\
\hline$a$ & 0.4403 & 0.8665 & 1.4321 & 3.0828 & 13.2832 & 0.9009 \\
$b$ & 2.7153 & 3.2379 & 4.7170 & 6.9677 & 8.4014 & 0.0001 \\
$R$ square & 0.8598 & 0.8280 & 0.8392 & 0.8115 & 0.5056 & -45.7899 \\
Adjusted $R$ & 0.8511 & 0.8172 & 0.8291 & 0.7998 & 0.4747 & 0.8178 \\
$P$ value & 0.0011 & 0.0015 & 0.0032 & 0.0031 & 0.0009 & 0.8064 \\
\hline
\end{tabular}

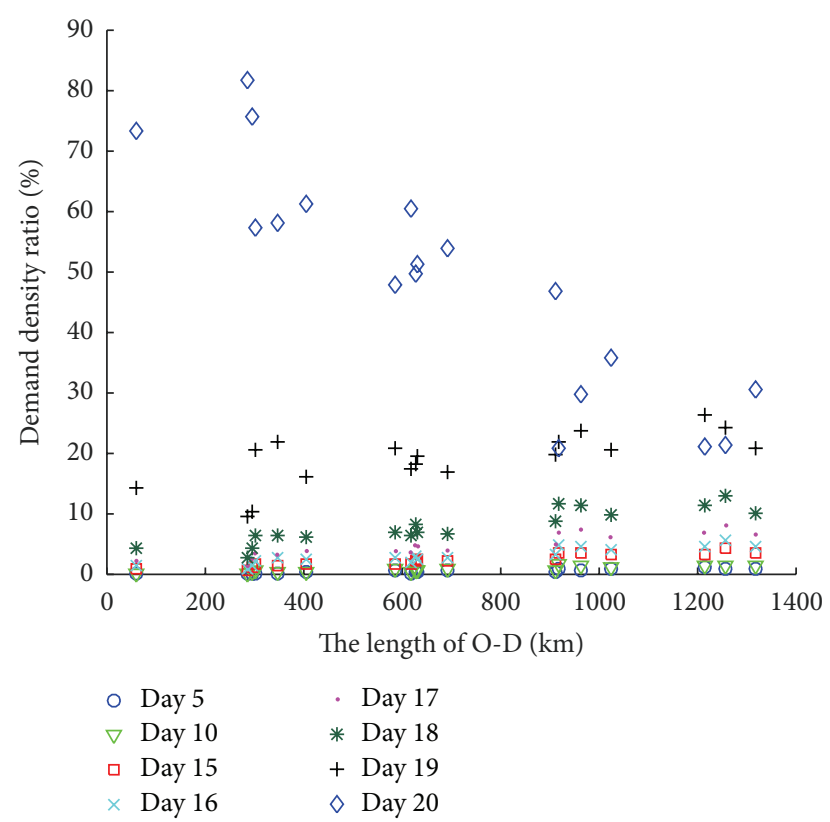

FIGURE 2: Correlation between demand density ratio and length of OD.

For a certain train $w$, let $d_{s}^{w, k}$ denote hourly demand density of its OD pair $k$ on day $s$. The total booking horizon is $S$ days; then the ratio of demand density on day $s$ is $y_{s}^{w, k}=$ $\left(d_{s}^{w, k} / \sum_{s=1}^{S} d_{s}^{w, k}\right) \times 100 \%$. Averaging the value $y_{s}^{w, k}$ of all the trains yields the daily demand density ratio of OD pair $k$ on day $s$, which is denoted as $y_{s}^{k}$. Figure 2 shows the relationship between daily demand density ratio and the length of the OD arc. The correlation between these is positive before day 18 , not significant on day 19 , and negative on the departure day.
From the figure, it appears that the daily demand density ratio $(y \%)$ and the length of OD pair $(1000 x)$ have a linear relationship. A linear regression equation was developed to test this hypothesis, which is $\hat{y}=\widehat{a}+\widehat{b} x$. Table 1 shows the regression analysis results, with the confidence coefficient $95 \%$.

The regression analysis results are revealing. The $P$ value is less than 0.05 for all days. The $R$-Square value is greater than 0.7 on days except for $1,6,7$, and 19 . Thus, there is a weaker linear relation between demand density ratio and OD length for these four days, though a fairly strong linear relation for the other days. The results also reveal some passenger arrival trends. Not considering other factors, passengers with long-distance journeys tend to purchase tickets early in the booking horizon, whereas short-distance journey passengers purchase tickets later. The slope of $x$ on the departure day is much greater than for other days, illustrating that the shorter the journey is, the more passengers are willing to purchase tickets just before departure.

(2) The Amount of Alternative Products. The other factor influencing passenger behavior is the number of trains that serve the same OD. We term these services alternative products. The more the alternative products exist, the later passengers purchase tickets. This conjecture can be realistic because if there are enough alternative products, passengers do not have to buy tickets in advance for the sake of tickets being not enough, and instead they will purchase tickets after everything of the travel is settled down.

To examine this, we selected two similar length pairs of ODs and used their ticket data to analyze the relationship between demand density ratio and amount of alternative products. Figure 3 compares the demand density ratio curves for itineraries of these two OD pairs. The booking horizon 


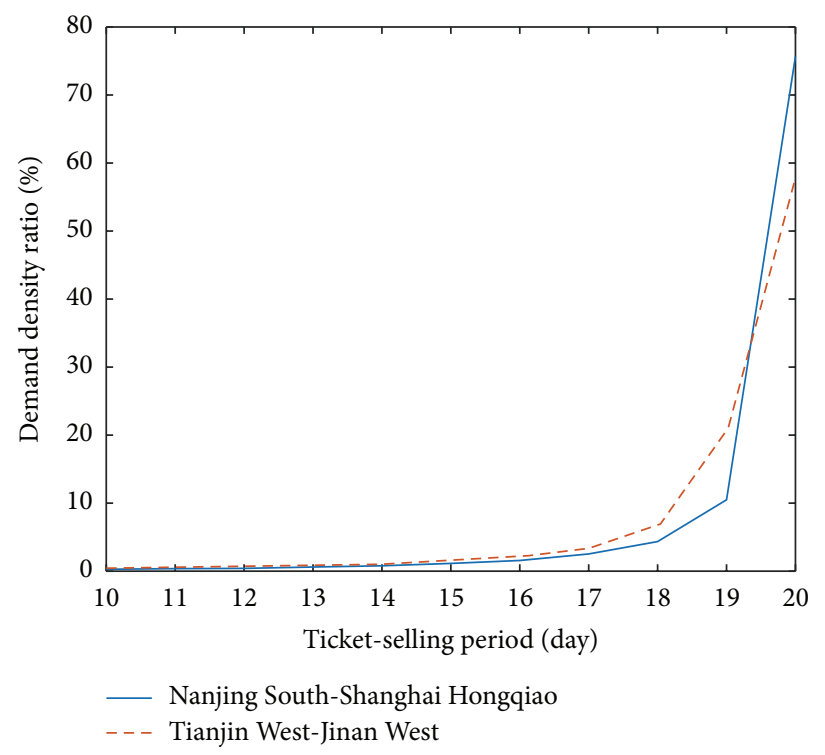

(a)

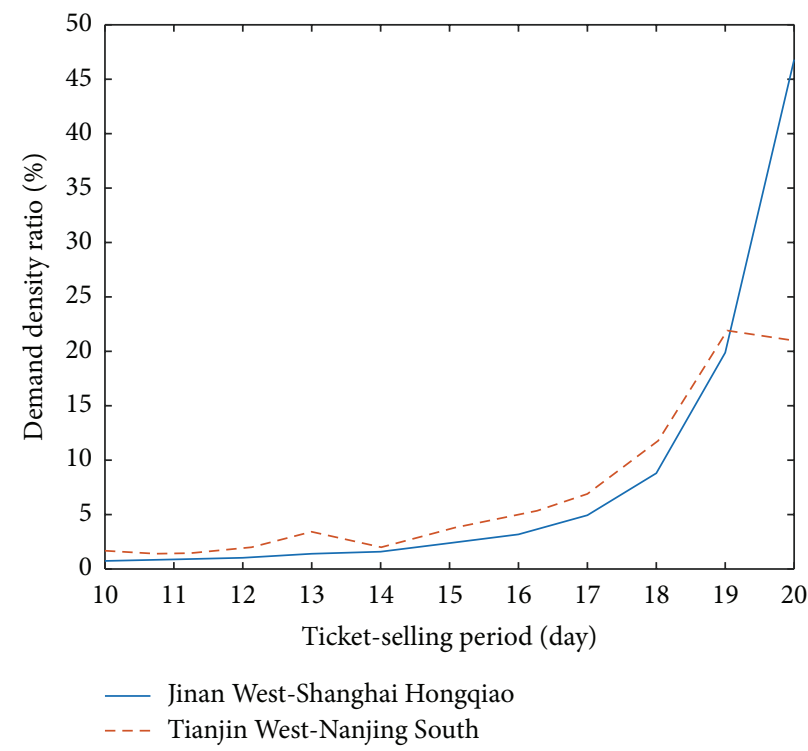

(b)

FIGURE 3: Relationship between demand density ratio and amount of alternative products.

of the ticket data we used in this examination is 20 days. Since the curves for days 1 to 9 are basically the same, the figure shows only the curves from days 10 to 20. Figure 3(a) represents the Nanjing South-Shanghai Hongqiao itinerary, where the length is $295 \mathrm{~km}$ and there are 44 alternative products, and the Tianjin West-Jinan West itinerary $(301 \mathrm{~km}$ and 4 alternate products). Figure 3(b) reflects the Jinan WestShanghai Hongqiao (912 km and 42 alternate products) and Tianjin West-Nanjing South $(918 \mathrm{~km}$ and 4 alternate products) itineraries.

In both Figures 3(a) and 3(b), before day 19 the curve of the OD with more alternative products lies above that of the OD with fewer products. On day 20, the reverse is true. We conclude that, for ODs with similar length, passengers purchase tickets earlier when there are fewer alternative products.

(3) The Purpose of the Trip. We can see from the statistical result of "Beijing-Shanghai High-Speed Railway demand questionnaire" (carried out by Beijing-Shanghai High-Speed Railway Company in 2012) that business travelers usually purchase tickets 1-2 days ahead of departure day, while the passengers traveling for tour, visiting friends, and migrant work tend to purchase tickets more than 3 days in advance. Thus it is convincing to assume that customers who are more price-sensitive arrive earlier and the less price-sensitive customers arrive later, which has already been commonly adopted by airline revenue management.

(4) The Train Departure Time. From Figure 1 we can see that in the earlier stage of ticket-sale period G1 curve is above G3 curve most of the time and only in the last day G1 curve is below G3 curve, which means that G1 passengers tend to purchase tickets earlier than G3 passengers. From this comparison we cannot arbitrarily make the conclusion that the earlier the departure time is, the earlier passengers purchase tickets. But the departure time of the train indeed has some influence on passenger behavior, which still needs more intensive study.

3.2. The Segmentation of Ticket-Sale Period. The passenger arrival regularity varies from train to train, even from section to section for a certain train, for the passenger behavior is influenced a lot by the train operation properties such as departure time and travel time. Therefore, the segmentation of ticket-sale period should be treated differently by each train and by each section for a given train.

The experience of Britain and France railway shows that too many price classes may cause the revenue management lose efficacy [17-19]. Thus in accordance with the statistical passenger arrival regularity, we plan to set two price classes for each product and divide the ticket-sale period into two subperiods; that is, for the product of Beijing-Nanjing and Beijing-Shanghai transportation service from the first day to 8th day is number 1 subperiod and from 9th day to 10th day is number 2 subperiod; as regards Nanjing-Shanghai product, the first day to 9 th day belong to number 1 subperiod, and 10th day itself is number 2 subperiod.

3.3. The Analysis of Computation Result. We take G3 train as an example here to examine our ticket fare optimization method. According to the modeling approach discussed in Section 2.1, G3 train can be described as a network shown in Figure 4.

For the network in Figure 2, a relation matrix of resource and product is created:

$$
a_{m n}=\left[\begin{array}{lll}
a_{11} & a_{12} & a_{13} \\
a_{21} & a_{22} & a_{23}
\end{array}\right]=\left[\begin{array}{lll}
1 & 1 & 0 \\
0 & 1 & 1
\end{array}\right] .
$$


TABLE 2: Reference price strategy and the revenue.

\begin{tabular}{lcccccc}
\hline \multirow{2}{*}{ Product } & Price (yuan) & \multicolumn{2}{c}{ Price elasticity of } & \multicolumn{2}{c}{ Demand density } & \multicolumn{2}{c}{ Duration (day) } \\
& $p_{0}$ & $\epsilon_{0}^{1}$ & $\epsilon_{0}^{2}$ & $\lambda_{0}^{1}$ & $\lambda_{0}^{2}$ & $l^{1}$ \\
\hline Beijing-Nanjing & 443.5 & 1.3 & 1.1 & 24 & 113 & 8 \\
Beijing-Shanghai & 553 & 1.3 & 1.1 & 45 & 148 & 8 \\
Nanjing-Shanghai & 134.5 & 1.3 & 1.1 & 7 & 290 & 9 \\
\hline Passenger traffic & & & & & & \\
Revenue (yuan) & & & 524,337 & & \\
\hline
\end{tabular}

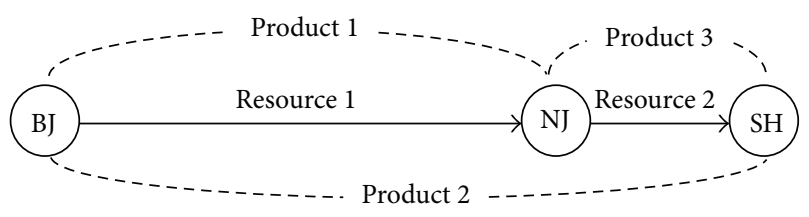

FIGURE 4: The network of G3 train.

The vehicle model of G3 train is CRH380BL, of which the personnel quota is 1005 . Thus, the resource capacity in our model is $x^{1}=x^{2}=1005$. As to the preallocation plan, we do not take standing-room ticket and overbooking into consideration. The product capacity in this case is set fixed; that is, $y^{1}=y^{3}=405, y^{2}=600$. The ticket-sale period of G3 train is divided as discussed in Section 3.2. However, the 10th day is departure day, for which the actual ticketsale time is not a whole day. The durations of each product in each subperiod for calculation in the case are shown in Table 1. As discussed above, the passengers arriving in the first subperiod are more price-sensitive than passengers arriving in the second subperiod. Thus we set the value of $\epsilon_{0}$ higher for the first subperiod and lower for the second, which can also be seen in Table 2.

A common demand function of a product can describe the quantitative relation between product demand and its impact factors, for example, product attributes (such as price) or marketing variables (such as advertising, brand name, and competitor's prices). From the consideration of research emphasis, we just focus on how the demand changes with product price without considering other impact factors. The Log-linear demand function is adopted in our study, which is popular in econometric studies and has several desirable theoretical and practical properties [20]. For example, the demand is always nonnegative so we can treat price unconstrained in the model. Besides that, the Log-linear function can be translated into a linear form by taking the log of the function; thus it can be estimated using linear regression. The demand-price function we use is as below:

$$
\lambda(p)=\lambda_{0} e^{-\epsilon_{0}\left(p / p_{0}-1\right)}
$$

In function (8), $p_{0}$ is interpreted as a reference price, $\lambda_{0}$ is the demand rate (the number of tickets sold per day) at the reference price, and $\epsilon_{0}$ is the absolute value of price elasticity of demand at the reference price. The negative sign in front
TABLE 3: Calculation results for the case.

\begin{tabular}{lcccc}
\hline \multirow{2}{*}{ Product } & \multicolumn{2}{c}{ Price (yuan) } & \multicolumn{2}{c}{ Demand density } \\
& $p^{1}$ & $p^{2}$ & $\lambda^{1}$ & $\lambda^{2}$ \\
\hline Beijing-Nanjing & 390.9 & 447.1 & 28 & 112 \\
Beijing-Shanghai & 525.5 & 599.2 & 48 & 135 \\
Nanjing-Shanghai & 108.5 & 122.5 & 9 & 320 \\
\hline Passenger traffic & & & 1267 & \\
Revenue (yuan) & & & 528,331 & \\
\hline
\end{tabular}

of $\epsilon_{0}$ means the demand and price are antidependent. This Log-linear demand function has a unique inverse, which is the price function:

$$
p(\lambda)=\left(\epsilon_{0}^{-1} \ln \left(\frac{\lambda_{0}}{\lambda}\right)+1\right) p_{0} .
$$

Matlab R2014a is applied for implementing our algorithm. The calculation results including resulting prices, demand densities, and the revenue to be gained using this price strategy are shown in Table 3.

In Table $3, p^{1}$ and $p^{2}$ represent the price of each product in the first and the second subperiod, respectively. $\lambda^{1}$ and $\lambda^{2}$ are the relevant demand density at new price in these two subperiods. From the solution we can see that, for a certain product, the price in the first subperiod is lower than that in the second subperiod, which just accords with the core idea of revenue management that to provide proper price for proper customer. Compared with the original pricing strategy, our floating pricing strategy can increase the revenue of the train by $0.76 \%$, which is $¥ 3994$. Right now there are 284 trains operated on Beijing-Shanghai High-Speed Railway per day. The total revenue will have a huge increase of about one million yuan even if each train's revenue just increases by $¥ 3994$. Our example also exhibits the demand densities of all the three products in subperiod 1 increase, and those in the subperiod 2 decrease compared with the demand densities under reference price, which proves our floating price strategy also plays a part in regulating demand.

\section{Conclusion}

We have shown how to optimize ticket fare for single highspeed train. Compared with the trial of Beijing-Shanghai High-Speed Railway Company, our proposal price strategy 
sets prices separately by OD and time period. An optimization model and corresponding algorithm were designed to solve the multiple fare issue. The difference between our model and other relevant ones is that it takes product capacity constraint into consideration. In the end, an experimental study is carried out to verify the method proposed previously. We take the ticket-sale data of G3 train in 2012 as an example. The case results show that our pricing strategy can not only increase the train revenue but also regulate the existing demand and stimulate the potential demand.

There are certainly many possible directions for future research in this area. We will next expand our study to multiple trains, considering the passengers' choice behavior among these train services. Another research direction is to dynamically determine when to open each fare grade based on the available seats left and time left.

\section{Competing Interests}

The authors declare that they have no competing interests.

\section{Acknowledgments}

This work is supported by China Railway Corporation Technology Research and Development Plan Project (2016X005E).

\section{References}

[1] L. R. Weatherford and S. E. Bodily, "A taxonomy and research overview of perishable-asset revenue management: yield management, overbooking, and pricing," Operations Research, vol. 40, no. 5, pp. 831-844, 1992.

[2] J. I. McGill and G. J. Van Ryzin, "Revenue management: research overview and prospects," Transportation Science, vol. 33, no. 2, pp. 233-256, 1999.

[3] G. Bitran and R. Caldentey, "An overview of pricing models for revenue management," Manufacturing \& Service Operations Management, vol. 5, no. 3, pp. 203-229, 2003.

[4] W. Elmaghraby and P. Keskinocak, "Dynamic pricing in the presence of inventory considerations: research overview, current practices, and future directions," Management Science, vol. 49, no. 10, pp. 1287-1309, 2003.

[5] A. Armstrong and J. Meissner, "Railway revenue management: overview and models," Working Paper, Lancaster University Management School, Lancaster, UK, 2010.

[6] R. Preston McAfee and V. te Velde, "Dynamic pricing in the airline industry," Working Paper, California Institute of Technology, Pasadena, Calif, USA, 2005.

[7] W. M. Kincaid and D. A. Darling, "An inventory pricing problem," Journal of Mathematical Analysis and Applications, vol. 7, pp. 183-208, 1963.

[8] R. E. Chatwin, "Optimal dynamic pricing of perishable products with stochastic demand and a finite set of prices," European Journal of Operational Research, vol. 125, no. 1, pp. 149-174, 2000.

[9] G. Gallego and G. V. Ryzin, "Optimal dynamic pricing of inventories with stochastic demand over finite horizons," Management Science, vol. 40, no. 8, pp. 999-1020, 1994.
[10] G. Gallego and G. Van Ryzin, "A multiproduct dynamic pricing problem and its applications to network yield management," Operations Research, vol. 45, no. 1, pp. 24-41, 1997.

[11] Y. Feng and G. Gallego, "Optimal starting times for end-ofseason sales and optimal stopping times for promotional fares," Management Science, vol. 41, no. 8, pp. 1371-1391, 1995.

[12] F. Shi, "Optimal dynamic pricing of railway passenger tickets," Journal of the China Railway Society, vol. 24, no. 1, pp. 1-4, 2002.

[13] X. Zhang, Research on Railway Ticket Discount Sales in China, Southwest Jiaotong University, Sichuan, China, 2005.

[14] X. Zhang, W. Luan, Q. Cai, and B. Zhao, "Research on dynamic pricing between high speed rail and air transport under the influence of induced passenger flow," Information Technology Journal, vol. 11, no. 4, pp. 431-435, 2012.

[15] E. Yao, Q. Yang, Y. Zhang, and X. Sun, "A study on high-speed rail pricing strategy in the context of modes competition," Discrete Dynamics in Nature and Society, vol. 2013, Article ID 715256, 6 pages, 2013.

[16] Q. Bingyi, Research on revenue management for dedicated passenger line based on passenger choice behavior [Ph.D. thesis], Southwest Jiaotong Universiy, 2014.

[17] K. Xiang'an, TGV High-Speed Railway in France, Southwest Jiaotong University Press, Chengdu, China, 1997.

[18] I. Abe, Revenue Management in the Railway Industry in Japan and Portugal: A Stakeholder Approach, Massachusetts Institute of Technology (MIT), Cambridge, Mass, USA, 2007.

[19] J. Preston, "Competition in British railways-what have we learned," in Proceedings of the Danish Transport Conference, Aalborg, Denmark, August 1999.

[20] K. T. Talluri and G. J. van Ryzin, The Theory and Practice of Revenue Management, International Series in Operations Research \& Management Science, Kluwer Academic, Boston, Mass, USA, 2004. 


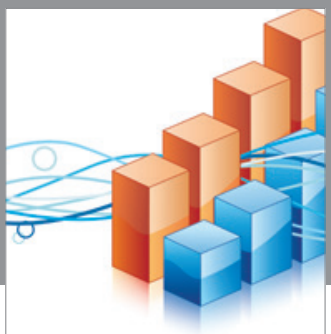

Advances in

Operations Research

vatem alat4

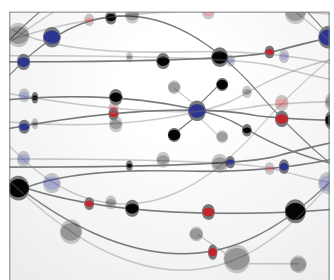

\section{The Scientific} World Journal
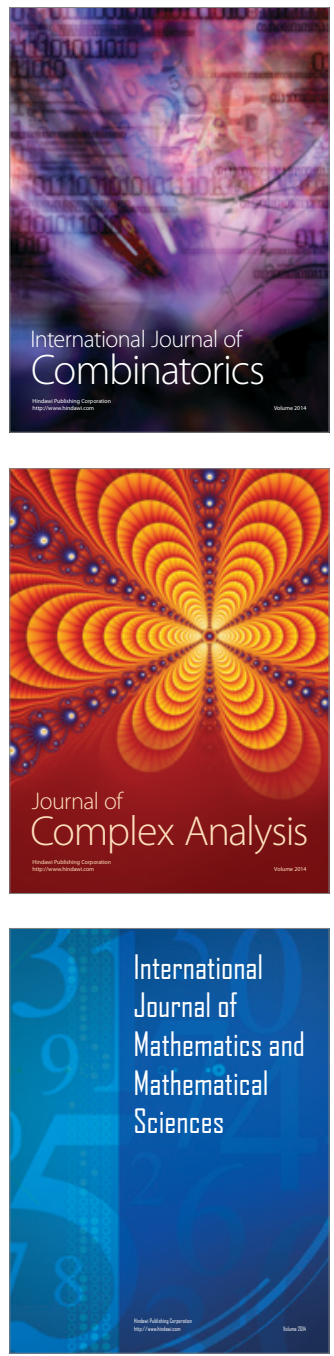
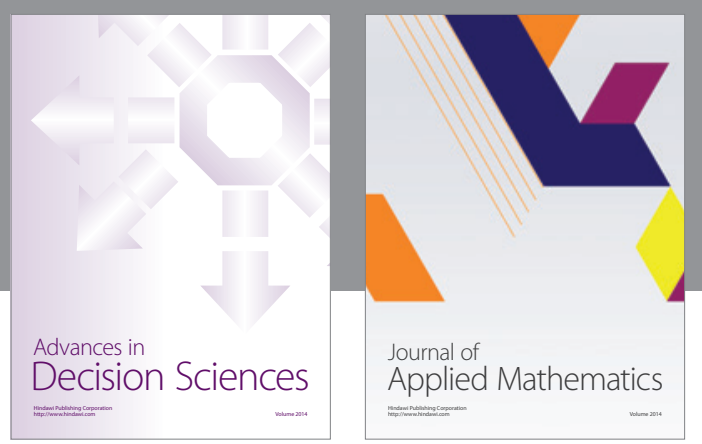

Algebra

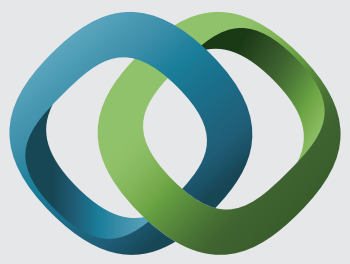

\section{Hindawi}

Submit your manuscripts at

http://www.hindawi.com
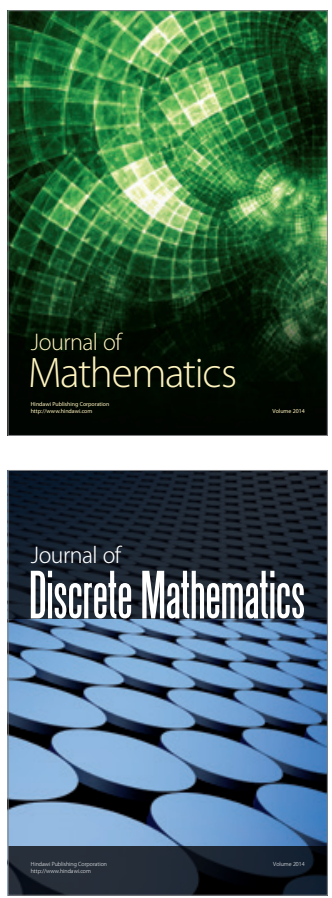

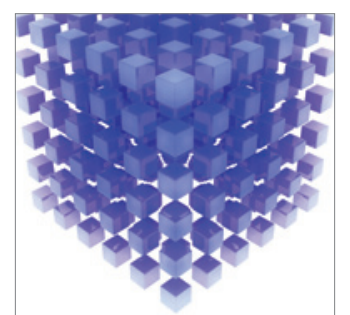

Mathematical Problems in Engineering
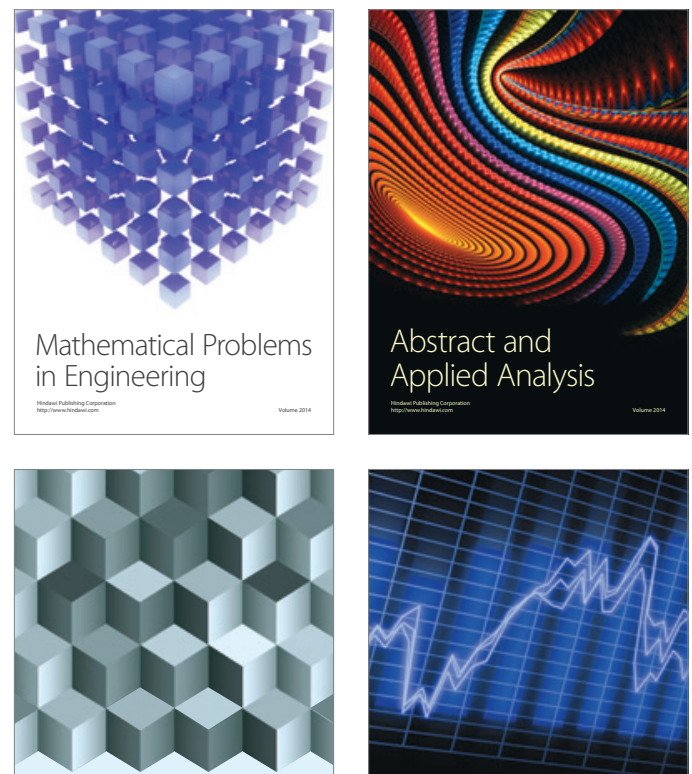

Journal of

Function Spaces

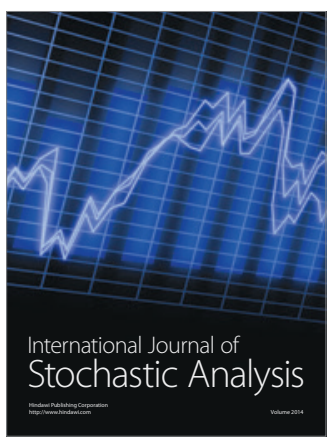

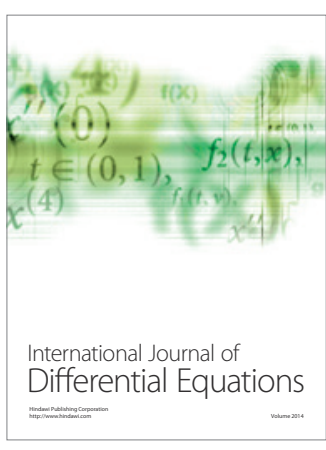
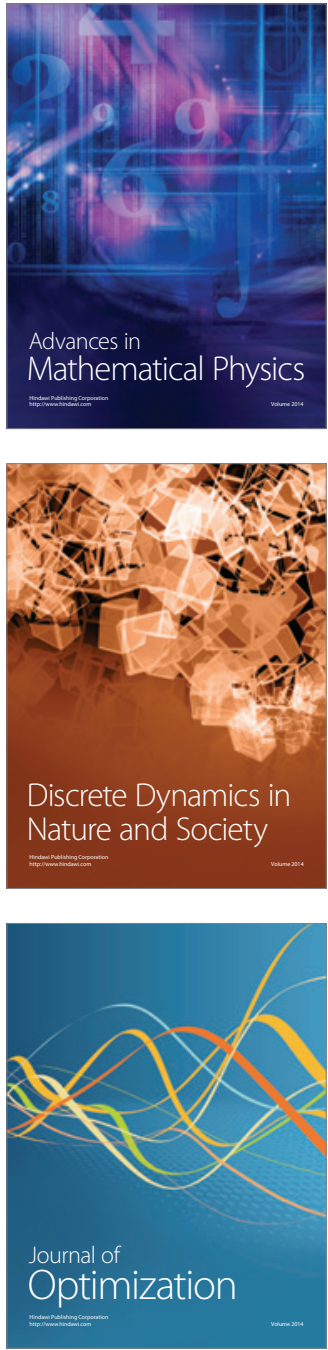